\title{
The ventral habenulae of zebrafish develop in prosomere 2 dependent on Tcf7l2 function
}

\author{
Carlo A Beretta', Nicolas Dross ${ }^{2}$, Peter Bankhead ${ }^{2}$ and Matthias Carl ${ }^{1 *}$
}

\begin{abstract}
Background: The conserved habenular neural circuit relays cognitive information from the forebrain into the ventral mid- and hindbrain. In zebrafish, the bilaterally formed habenulae in the dorsal diencephalon are made up of the asymmetric dorsal and symmetric ventral habenular nuclei, which are homologous to the medial and lateral nuclei respectively, in mammals. These structures have been implicated in various behaviors related to the serotonergic/dopaminergic neurotransmitter system. The dorsal habenulae develop adjacent to the medially positioned pineal complex. Their precursors differentiate into two main neuronal subpopulations which differ in size across brain hemispheres as signals from left-sided parapineal cells influence their differentiation program. Unlike the dorsal habenulae and despite their importance, the ventral habenulae have been poorly studied. It is not known which genetic programs underlie their development and why they are formed symmetrically, unlike the dorsal habenulae. A main reason for this lack of knowledge is that the $\mathrm{vHb}$ origin has remained elusive to date.

Results: To address these questions, we applied long-term 2-photon microscopy time-lapse analysis of habenular neural circuit development combined with depth color coding in a transgenic line, labeling all main components of the network. Additional laser ablations and cell tracking experiments using the photoconvertible PSmOrange system in GFP transgenic fish show that the ventral habenulae develop in prosomere 2, posterior and lateral to the dorsal habenulae in the dorsal thalamus. Mutant analysis demonstrates that the ventral habenular nuclei only develop in the presence of functional Tcf 72 , a downstream modulator of the Wnt signaling cascade. Consistently, photoconverted thalamic $t c f 7 / 2^{\text {exl/exl }}$ mutant cells do not contribute to habenula formation.

Conclusions: We show in vivo that dorsal and ventral habenulae develop in different regions of prosomere 2 . In the process of ventral habenula formation, functional tcf $7 / 2$ gene activity is required and in its absence, ventral habenular neurons do not develop. Influenced by signals from parapineal cells, dorsal habenular neurons differentiate at a time at which ventral habenular cells are still on their way towards their final destination. Thus, our finding may provide a simple explanation as to why only neuronal populations of the dorsal habenulae differ in size across brain hemispheres.
\end{abstract}

Keywords: Habenula, Tcf, Zebrafish, Time-lapse imaging, Photoconversion, PSmOrange, Neural network, Asymmetry

\section{Background}

The habenulae are part of the conserved dorsal diencephalic conduction system and connect the forebrain with the ventral mid- and hindbrain via axon bundles named fasciculi retroflexi [1]. In teleosts, these efferent axons derive from the bilateral dorsal $(\mathrm{dHb})$ and ventral habenular $(\mathrm{vHb})$ nuclei, which innervate the interpeduncular nucleus (IPN) and the median raphe (MR), respectively [2-5]. Consistent

\footnotetext{
* Correspondence: matthias.carl@medma.uni-heidelberg.de

${ }^{1}$ Department of Cell and Molecular Biology, Medical Faculty Mannheim, Heidelberg University, Ludolf-Krehl-Strasse 13-17, Mannheim 68167, Germany Full list of author information is available at the end of the article
}

with the function of network components in the regulation of a number of neurotransmitters, the habenulae have been implicated in a range of behaviors and cognitive functions, and aberrant habenular network function has been correlated with pathophysiological syndromes such as depression and schizophrenia in mammalian model systems and humans [6-10]. An additional intriguing hallmark of the system is the distinct asymmetric character of the $\mathrm{dHb}$ across the left-right axis in many vertebrates with respect to neuroanatomy, expression of various molecules and connectivity patterns $[3,4,11-14]$. Also in mammals, subtle size differences between the left and right medial habenulae, the

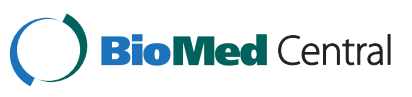


structure homologous to the $\mathrm{dHb}$ of teleosts [2], have been described [15]. In particular these asymmetric features have led to researchers investigating this part of the habenulae as a model for functional lateralization of the brain, common to all vertebrates $[16,17]$. To assess this fundamental aspect in neuroscience, a considerable amount of work has been dedicated to elucidating the genetic cascades underlying asymmetric $\mathrm{dHb}$ development, to be able to manipulate the network and study the consequences $[11,18,19]$. In contrast, the analysis of $\mathrm{vHb}$ development has been largely neglected. For instance, it is known that $\mathrm{dHb}$ neurons differentiate from pools of precursor cells adjacent to the pineal complex on the left and right [20], but nothing is known about the origin of the $\mathrm{vHb}$. This, however, comes as a surprise as the lateral habenulae, the mammalian part of the habenulae homologous to the $\mathrm{vHb}$ in teleosts [2], is a prominent component of the serotonergic system modulating for instance aggressive behavior [21-23]. Moreover, this part of the habenulae was found to be a key-regulator of the reward system [24-27]. Interestingly, the lateral habenulae in albino mice exhibit a right-sided lateralization [28], but the functional importance has not been reported.

To find out about $\mathrm{vHb}$ origin and development, we used 2-photon microscopy time-lapse imaging and followed habenular neural circuit development for four consecutive days in transgenic zebrafish embryos. We found evidence that $\mathrm{vHb}$ neurons originate in the thalamic-epithalamic part of prosomere 2, and during development come to lie adjacent to the forming dorsal habenulae. We validated our observation by laser cell ablations and by tracking cells using the photoconvertible protein PSmOrange [29] in the GFP transgenic background. This system also allowed us to demonstrate that $\mathrm{vHb}$ neuron development crucially relies on the Wnt pathway downstream modulator Tcf7l2.

\section{Results and discussion}

Long-term imaging suggests the intermingling of habenular neurons derived from different clusters in the dorsal diencephalon

All habenular nuclei and their efferent projections targeting the interpeduncular nucleus (IPN) and median raphe (MR) are labeled in Et(-1.0otpa:mmGFP)hd1 transgenic embryos (unpublished data). In addition to GFP expression starting at $43 \mathrm{hpf}$ in developing dorsal habenular $(\mathrm{dHb})$ neurons in close proximity to $c x c r 4 b$ expressing habenular precursor cells ([20] and unpublished data), a bilateral cell cluster in prosomere 2 (hereafter named thalamic-epithalamic early projecting cluster or ThEPC), posterior and lateral to them, expresses GFP (Figure 1a,a'). Measuring the distance of ThEPCs and $\mathrm{dHb}$ neurons at $48 \mathrm{hpf}$ in 2D, we find them separated by about $40 \mu \mathrm{m}$. Performing long-term 2-photon microscopy (2-PM) high-resolution imaging of habenular neural circuit development combined with depth color coding, we noticed that the most anterior ThEPC cells appear to come to lie adjacent to $\mathrm{dHb}$ neurons over time in a lateral position (Figure 1a-a"' and Additional file 1: Movie S1). This part of the established habenulae has previously been shown to contain ventral habenula $(\mathrm{vHb})$ neurons at this developmental stage (Figure 2a) [2]. To find out more about the ThEPC cell composition, we imaged ThEPC development in Et(-1.0otpa:mmGFP)hd1 transgenic embryos with high magnification, starting before the onset of GFP expression in $\mathrm{dHb}$ cells at 43 hours post fertilization (hpf) (Additional file 2: Movie S2). While some ThEPC neurons project axons (Figure 1b and Additional file 2: Movie S2), some other cells of this cluster still divide between 48 and $52 \mathrm{hpf}$ (Figure 1b-b"). Intriguingly, we found that a number of ThEPC neurons express the serotonergic marker $5 \mathrm{HT}$ at 2 days post fertilization (dpf), suggesting that the clusters consist of mixed cell populations (Figure 1c-c"). vHb neurons are part of the serotonergic system, which exhibits a striking asymmetry in salmon species [2,30,31]. Although we did not observe any differences in the number of $5 \mathrm{HT}$ expressing neurons across the brain at this developmental stage, our findings led us speculate that a subpopulation of thalamic neurons might contribute to the $\mathrm{vHb}$.

\section{Ventral and dorsal habenular neurons originate in different areas of prosomere 2}

To further support this idea, we unilaterally ablated the entire developing $\mathrm{dHb}$ in Et(-1.0otpa:mmGFP)hd1 transgenic embryos after the onset of GFP expression at $53 \mathrm{hpf}$ (Additional file 3: Figure S1a-b') and investigated GFP expression in the habenulae two days later. At this time, dHb cells and ThEPC cells can be well distinguished (Additional file 3: Figure S1a inset) and the missing IPN innervation by axons from the ablated $\mathrm{dHb}$ served as a control for their complete absence. If the origin of the $\mathrm{vHb}$ differs from the $\mathrm{dHb}$, we would expect to see some GFP positive neurons in the lateral part of the habenula on the ablated side. Indeed, two days after ablation, green neurons were detected in this area independent of the side of ablation (Figure 2a-c). For further evidence, we next conducted the converse experiment and unilaterally ablated one ThEPC at the onset of GFP expression in Et (-1.0otpa:mmGFP)hd1 transgenic embryos (Additional file 4: Figure S2). Consistent with our hypothesis that some ThEPC neurons contribute to the habenulae, this ablation resulted in the development of a smaller habenula on the ablated side compared to the habenula on the non-ablated side (Figure 2d,e).

Even though our time-lapse, marker analysis and ablation experiments strongly suggested that $\mathrm{dHb}$ and $\mathrm{vHb}$ spatial origins differ, we set out to further corroborate our finding by tracking thalamic cells over time. We used the photoconvertible fluorescent H2B-PSmOrange 


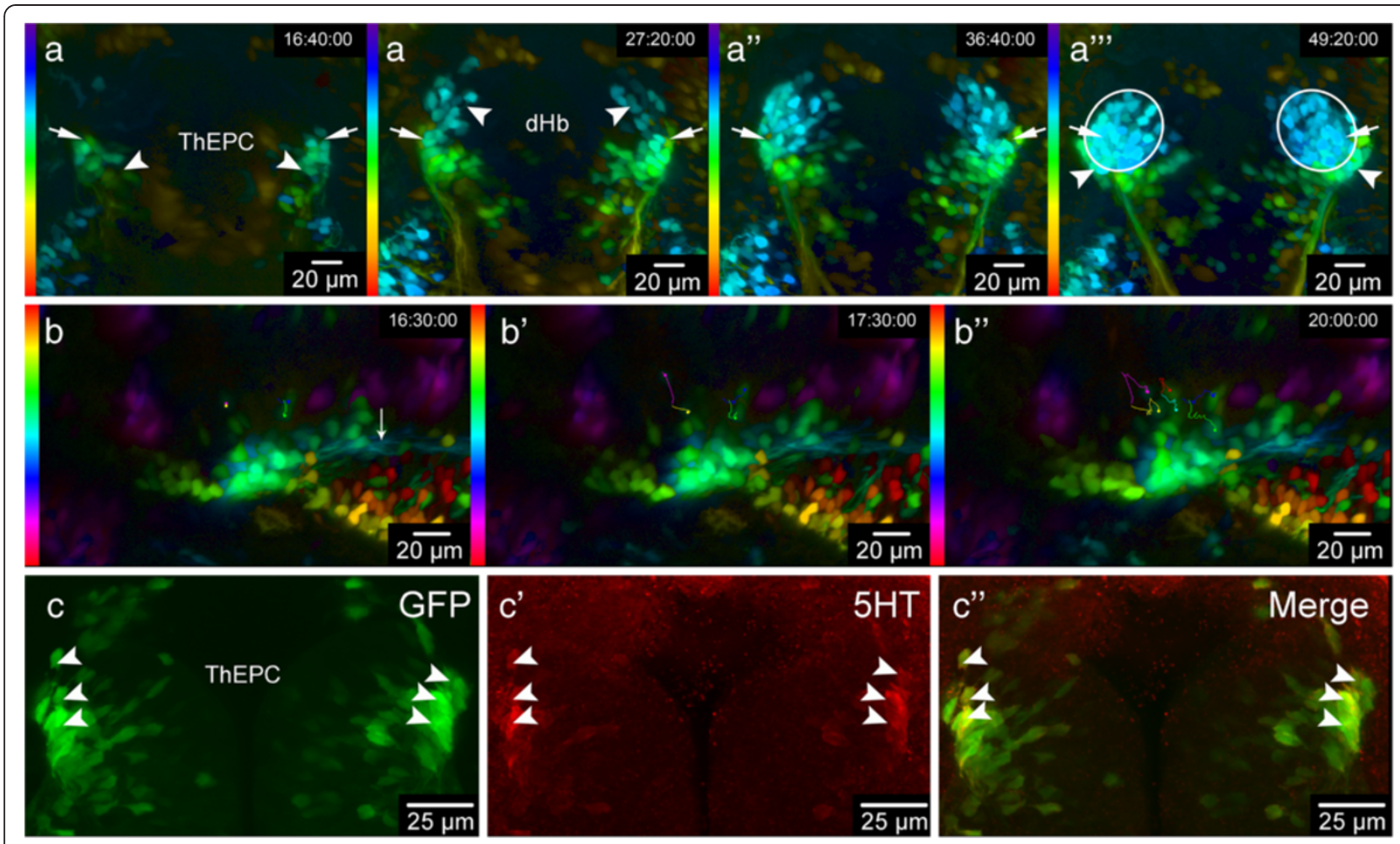

Figure 1 A subpopulation of thalamic-epithalamic early projecting cluster (ThEPC) cells may contribute to the habenulae. (a-a"') Color code MIP, dorsal views with anterior to the top of the habenula area in living Et(-1.00tpa:mmGFP)hd1 embryos at $46 \mathrm{hpf}, 57 \mathrm{hpf}, 68 \mathrm{hpf}$ and $79 \mathrm{hpf}$ (left to right; see Additional file 1: Movie S1). LUT (look up table) shows the z color code with a z-depth value of $300 \mu \mathrm{m}$. Arrows indicate the most anterior ThEPC cells, which appear to end up in the lateral part of the habenulae. (a) Arrowheads indicate the left and right ThEPCs. (a') Arrowheads indicate the left and the right dHb domain. (a"') Arrowheads highlight ThEPC cells, which remain outside the habenulae (circles). (b-b") Spectrum MIP, dorsal views with anterior to the left of left-sided ThEPC cells at $48 \mathrm{hpf}, 49 \mathrm{hpf}$ and $52 \mathrm{hpf}$ (see Additional file 2: Movie S2). LUT (look up table) shows the z spectrum with a z-depth value of $200 \mu \mathrm{m}$. Dots and lines indicate examples of manually tracked dividing cells. (b) Arrow indicates ThEPC axons. (c-c") Dorsal view of the thalamic area, MIP, anterior to the top, of a $48 \mathrm{hpf}$ Et(-1.0otpa:mmGFP)hd1 embryo stained for $5 \mathrm{HT}$. From left to right: GFP, red and merged channels. Arrowheads highlight some co-labeled GFP/5HT positive neurons. The gamma was adjusted to a value of 0.80 (b,c). $\mathbf{d H b}$, dorsal habenula; ThEPC, thalamic-epithalamic early projection cluster; MIP, maximum intensity projection.

protein, which changes its emission from orange to farred upon blue light treatment [29]. We find that the photoconverted protein is stable for at least three days in zebrafish and is an excellent tool for cell tracking in GFP transgenic fish (Figure 3a-c). We used the GFP positive ThEPC cells as a thalamic landmark and photoconverted the protein in, and closely around these cells at $2 \mathrm{dpf}$ (Figure 3a-c insets) and analyzed their position in the brain two days later (Figure $3 \mathrm{a}-\mathrm{c}$ ). Thus, cells carrying the photoconverted protein at $4 \mathrm{dpf}$ were derived from the thalamic area irrespectively of whether they co-expressed GFP. We developed an automated co-localization macro for ImageJ to unambiguously identify cells that express the photoconverted far-red fluorescent protein or co-express GFP and the photoconverted far-red fluorescent protein in the context of the habenular morphology highlighted by non-photoconverted orange fluorescent protein in the cell nuclei. We find on average 14 cells expressing the photoconverted protein in a vHb typical latero-ventral position in the habenulae (Figure 3a-c,f, Additional file 5:
Figure S3 and Additional file 6: Movie S3). On average, eight of these photoconverted cells co-expressed GFP and between five and eight GFP negative photoconverted cells were located in the $\mathrm{vHb}$ in their immediate vicinity $(\mathrm{n}=8)$. This indicated that unlike $\mathrm{dHb}$ neurons, which originate from precursor cells adjacent to the pineal complex on the left and right [20], some, if not all, $\mathrm{vHb}$ neurons originate from a more postero-lateral region of prosomere 2 (Figure 3d,e).

This finding may constitute a simple explanation as to why the $\mathrm{vHb}$ form symmetrically across the midline. The $\mathrm{dHb}$ consist of the lateral and the medial subnuclei, which are asymmetrically large in size between the left and right sides of the brain (Figure 2a). The differentiation of $\mathrm{dHb}$ precursor cells into neurons of these sub-nuclei is critically influenced by yet unknown signals from a group of parapineal cells, which during development migrate out from the anteriormost part of the pineal organ and are only present on the left side of the brain adjacent to developing $\mathrm{dHb}$ neurons [3,12,32-34]. 

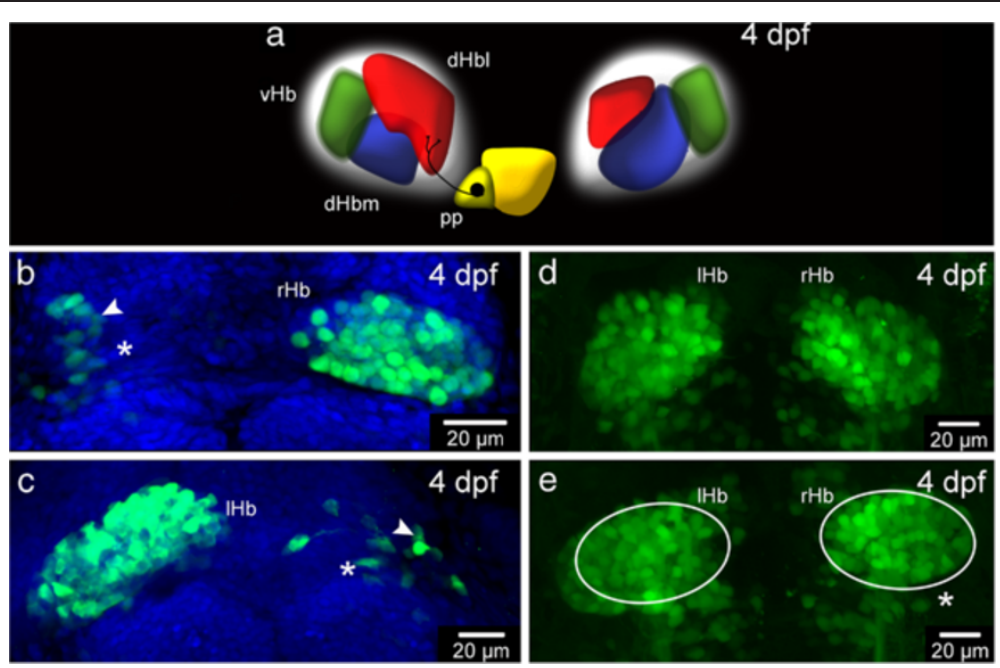

Figure 2 Ablation experiments suggest a contribution of thalamic-epithalamic early projecting cluster (ThEPC) cells to the habenulae. (a) Habenular nuclei at $4 \mathrm{dpf}$. dHbl, lateral dorsal habenula (red); dHbm, medial dorsal habenula (blue); vHb, ventral habenula (green); P, pineal (yellow); pp, parapineal (dark yellow). (b-e) MIP, anterior to the top, dorsal views of the habenular nuclei of $4 \mathrm{dpf}$ Et(-1.0otpa:mmGFP)hd1 embryos, after (b) left and (c) right $\mathrm{dHb}$ ablation at $2 \mathrm{dpf}$. Asterisks mark the ablated side and white arrowheads highlight neurons located in the lateral habenular domain. The nuclei (in blue) are labeled with Sytox Orange. (d,e) Habenula formation at 4 dpf in (d) not ablated and in (e) ThEPC ablated Et(-1.0otpa:mmGFP) hd1 embryos. Asterisk marks the ablated side. Same sized ellipses highlight the habenula size differences. The gamma was adjusted to a value of 0.80 . $\mathrm{IHb}$, left habenula; rHb, right habenula; ThEPC, thalamic-epithalamic early projecting cluster.

The removal of these parapineal cells between 24 and 28 $\mathrm{hpf}$ results in the formation of symmetric $\mathrm{dHb}$ due to an increased number of precursors differentiating into medial $\mathrm{dHb}$ neurons at the expense of lateral $\mathrm{dHb}$ neurons on the left side of the brain. At this time, the future $\mathrm{vHb}$ cells are still on their way towards their final destination and may therefore not receive any parapineal cell signals. Alternatively, $\mathrm{vHb}$ precursors could receive the parapineal cell signals, which, however, do not influence their differentiation program due to different intrinsic properties between $\mathrm{vHb}$ and $\mathrm{dHb}$ precursors. This scenario is less likely as the $\mathrm{dHb}$ progenitors, which are influenced by parapineal cell signals, are in close vicinity of the parapineal cells [11], suggesting that the signal is acting over only a relatively short distance.

\section{Tcf7l2 function is essential for ventral habenula development}

The upregulation of Wnt signaling in axin1 mutants [35] causes the formation of symmetric $\mathrm{dHb}$ with rightsided character similar to parapineal cell ablated embryos $[18,36]$. The parapineal cells often migrate to the left side of the mutant brains but their signaling cues do not influence $\mathrm{dHb}$ cell development in the presence of upregulated Wnt signaling. Thus, Wnt activity appears to influence the communication between pineal complex and $\mathrm{dHb}$. The involvement of Wnt signaling in $\mathrm{dHb}$ development and the established Wnt function in thalamus development [37-39] prompted us to investigate whether this pathway may also be involved in $\mathrm{vHb}$ development. However, we found that impaired Axin1 function does not affect the formation of the $\mathrm{vHb}$ as judged by the expression of the $\mathrm{vHb}$ cell marker kisspeptin-1 (kiss-1) [40] and the innervation of the $\mathrm{vHb}$ axon targets, the MR (Figure 4a-a"', b-b"').

To further elucidate the potential role of Wnt signaling in $\mathrm{vHb}$ development, we investigated embryos mutant for the Wnt signaling component $t c f 7 l 2$ [41], a gene widely expressed in the diencephalon [42]. Tcf7l2 exl/exl mutant embryos exhibit no morphological alterations for up to six weeks of development [41]. We find that kiss-1 expression is lost in $t c f 7 l 2^{\text {exl/exl }}$ mutants and the MR are not innervated by vHb axons (Figure 4a-a"', c-c"'). Moreover, photoconverted H2B-PSmOrange positive ThEPC cells did not localize in the $\mathrm{vHb}$ at $4 \mathrm{dpf}$ in the mutants (Figure 3a'-c, f' and Additional file 5: Figure S3). These data indicate that Tcf7l2 function is required for the formation of the $\mathrm{vHb}$. At the same time, our mutant analysis confirms the thalamic origin of $\mathrm{vHb}$ cells.

Tcf7l2 is a context dependent transcriptional downstream modulator of the Wnt signaling pathway [43]. However, in the light of our finding that only defective Tcf712 function and not impaired Axin 1 activity affects vHb development, we can only speculate that Tcf7l2 acts as an activator of Wnt signaling in the context of $\mathrm{vHb}$ development. In this scenario, only active Tcf712 mediated Wnt signaling but not the Axin1 mediated suppression of Wnt signaling is important for $\mathrm{vHb}$ development. 

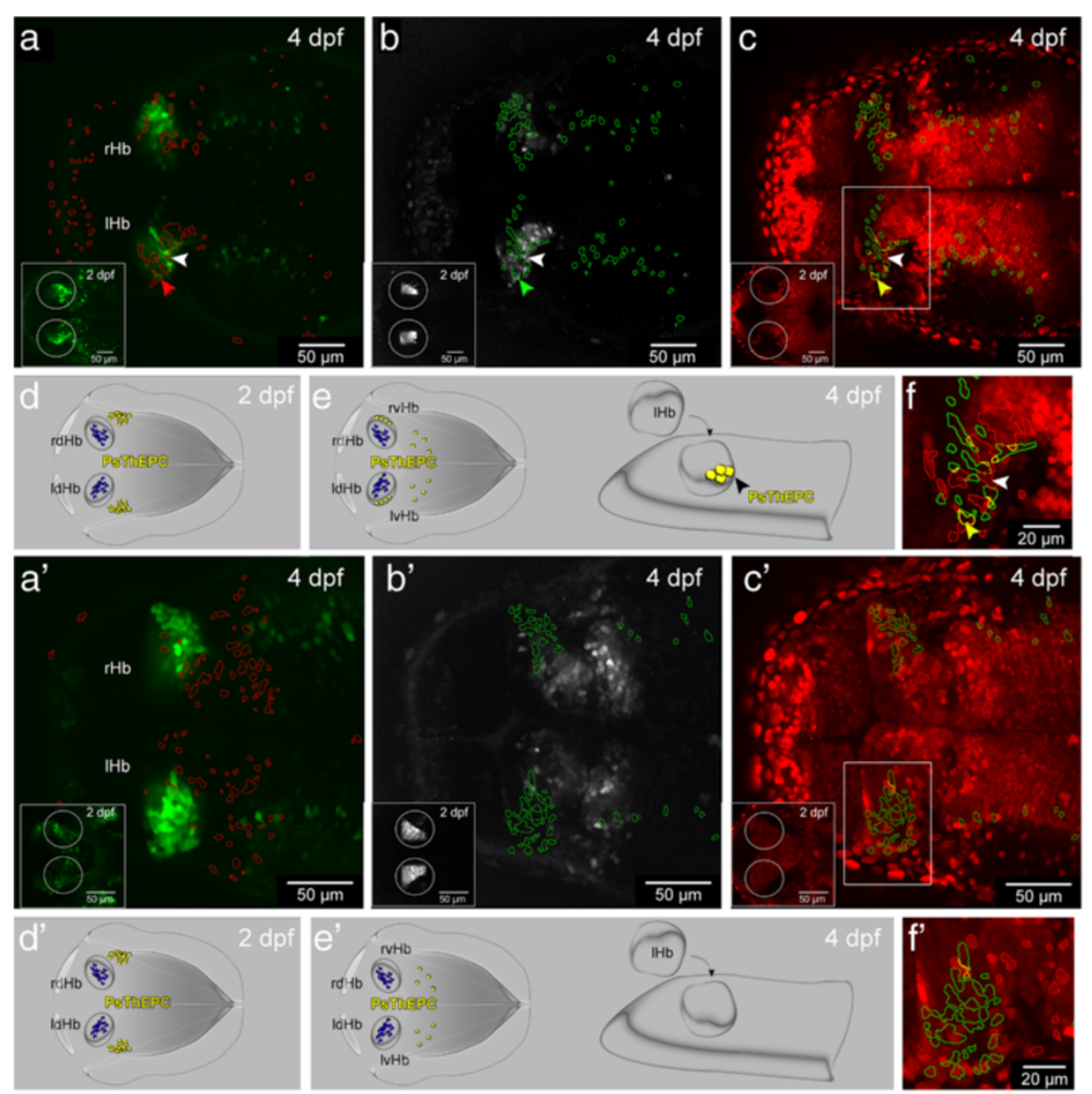

Figure 3 A population of thalamic cells forms the ventral habenula $(\mathbf{v H b})$ in the presence of Tcf7l2 function. (a-c') Dorsal views, anterior

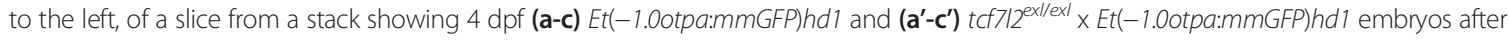
photoconversion of H2B-PSmOrange positive cells in the thalamus at $2 \mathrm{dpf}$ (insets). Circles highlight the area of photoconversion. All targeted cells express the photoconverted protein and are in the centre of the circles. The gamma was adjusted to a value between 0.60 and 0.90 . (a-a') Red ROls show the position of the photoconverted cells in the green channel. (b-b') Green ROls show the location of GFP positive cells in the far-red channel. (c-c') Red and green ROls were combined in the red channel to visualize the position of GFP photoconverted ThEPC neurons in the habenular nuclei in yellow. Box indicates the region shown in $\mathrm{f}$ and $\mathrm{f}^{\prime}$ with higher magnification for clarity. (a-c,f) Colored arrowheads mark a photoconverted ThEPC derived GFP positive cell in the habenula. White arrowheads mark a photoconverted GFP negative cell in the habenula. (a'-c', $\mathbf{f}^{\prime}$ ) Photoconverted cells are not found in the habenulae of tcf7/2 ${ }^{\text {ex/exl }}$ mutants. (d-e') Model showing the contribution of some ThEPC cells (yellow) to the vHb in wild type embryos but not in $t c f 7 / 2$ ex/exl mutants. $\mathrm{dHb}$ cells are marked in blue. IHb, left habenula; lvHb, left ventral habenula; Ps, photoconverted; rHb, right habenula; rvHb, right ventral habenula; $\mathrm{ROI}$, region of interest; ThEPC, thalamic-epithalamic early projecting cluster.

It is still an outstanding question as to which Wnt ligands are involved in habenula development. A number of ligands such as Wnt1, Wnt3a, Wnt7 genes and Wnt8b are expressed in, or in close proximity to, developing $\mathrm{dHb}$ neurons $[18,44]$ and may regulate the development of $\mathrm{dHb}$ neurons. Wnt3a is also expressed in the roof plate of prosomere 2 and influences the patterning and neurogenesis in the thalamus and the related thalamic mantle zone, which expresses downstream genes of Wnt signaling and harbors post-mitotic thalamic neurons [39]. It will be intriguing to determine whether indeed Wnt3a signaling influences vHb neuron development.

\section{Conclusions}

Using long-term time-lapse techniques, computational color coding of z-positions, laser cell ablations and cell tracking methods, we show that the $\mathrm{dHb}$ and $\mathrm{vHb}$ originate in different areas of prosomere 2. Furthermore, we identify $t c f 7 l 2$ as the first gene essential for vHb development. The $\mathrm{vHb}$ has important roles modulating the serotonergic system in vertebrates and its impairment in humans has various consequences ranging from mood fluctuations to schizophrenia and suicidal behavior [7,21]. Intriguingly, $t c f 7 l 2$ has also been linked to schizophrenia $[45,46]$ and in the light of our results it is 


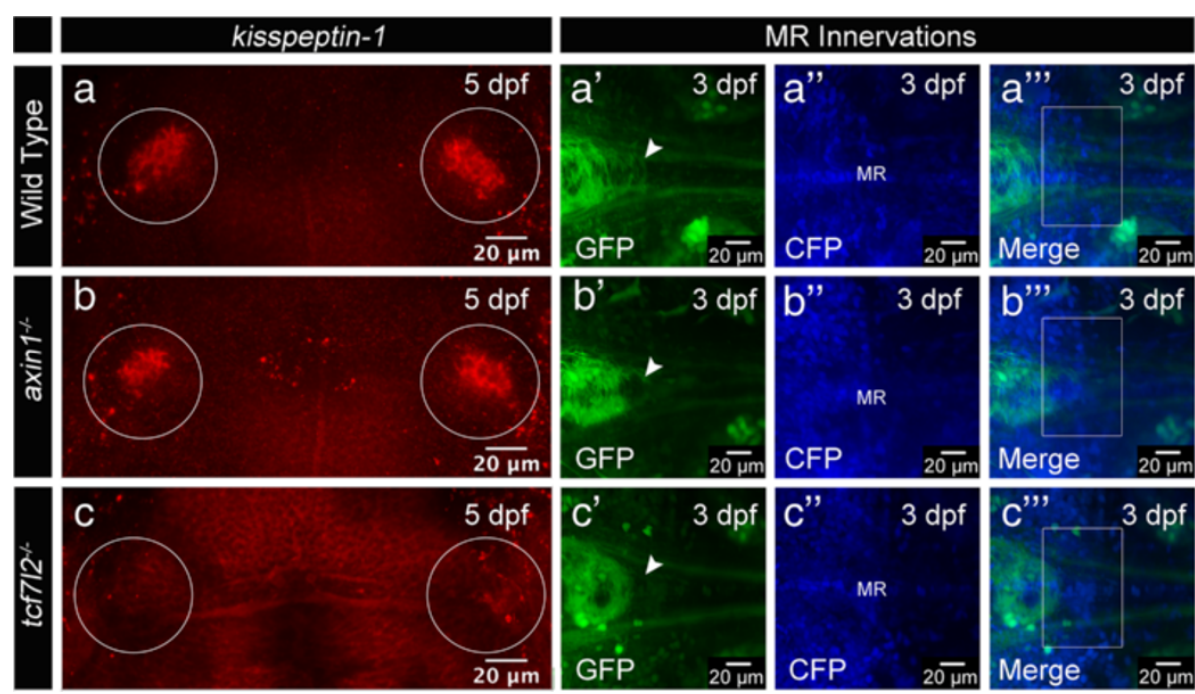

Figure 4 Tcf7l2 function is required for ventral habenula $(\mathbf{v H b})$ development. (a-c) Dorsal views, anterior to the top. kisspeptin-1 expression in the $\mathrm{vHb}$ (circle) is not affected by the upregulation of Wnt/beta-catenin signaling in (b) axin 1 mutant embryos, but absent in (c) tcf7/2 ex//exl mutants. (a'-c'"') MIPs, anterior to the left, dorsal views of axons innervating the MR in Et(1.0otpa:mmGFP)hd1 transgenic embryos. H2A-CFP labels

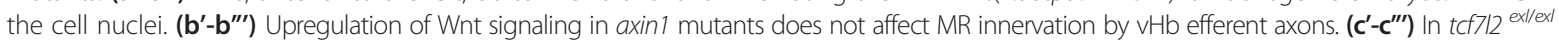
mutants, the MR are not innervated. White arrowheads and white boxes highlight the location of MR innervating axons, absent in ( $\left.\mathbf{c}^{\prime}-\mathbf{c}^{\prime \prime \prime}\right)$. The gamma was adjusted to values between 0.70 and 0.80 . vHb, ventral habenula; MR, median raphe.

tempting to speculate about a connection between Tcf7l2 function, vHb development and schizophrenia.

\section{Methods}

Fish maintenance

The zebrafish lines AB/TL, Et(-1.0otpa:mmGFP)hd1, tcf7l2 $2^{\text {exl/exl }}$ x Et(-1.0otpa:mmGFP)hd1 and axin1 $1^{\text {tm } 235} \mathrm{x}$ Et(-1.0otpa:mmGFP)hd1 were maintained and bred according to standard procedures [47]. All animal procedures were approved by the Regierungspräsidium Karlsruhe (permit AZ 35-9185.81/G-60/12). To inhibit pigmentation, embryos were incubated in $0.2 \mathrm{mM}$ PTU.

\section{tcf7/2 ${ }^{\text {exl/exl }} \times$ Et(-1.0otpa:mmGFP)hd1 genotyping}

Genomic DNA was extracted from tails of $t c f 7 l 2^{\text {exl/exl }} \mathrm{x}$ Et(-1.0otpa:mmGFP)hd1 incross derived embryos. A $t c f 7 l 2$ DNA fragment was amplified using the following primers: PrFw 5'-AAAATGCCGCAGCTGAAC-3' and PrRw 5'-CAACAACACGGTGCATCG-3'. The point mutated base pair was identified by BsajI digestion.

\section{Immunohistochemistry and in situ hybridization}

In situ hybridizations, antibody labelings and fluorescent in situ labelings were carried out as described [18,48-50]. Antibodies and fluophores used: rabbit anti-GFP (1:1000, Torrey Pines, CA, USA), Rabbit anti-5HT (1:1000, Sigma, Taufkirchen, Germany), Alexa Fluor 488 (1:250, Molecular Probes, Darmstadt, Germany), Alexa Fluor 647 (1:200, Molecular Probes, Darmstadt, Germany).
Whole mount in situ hybridization was performed with kiss-1.

The nuclei were labeled incubating embryos with Sytox Orange (1:100.000, Invitrogen, Darmstadt, Germany) in PBS, $0.8 \%$ Triton X-100, 1.0\% BSA for 30 minutes.

\section{2-PM laser ablation}

2-PM laser ablations of cells were performed using a Nikon 16x water dipping LWD objective (NA 0.80; Düsseldorf, Germany) on a multi photon LaVision BioTec TriM Scope (Bielefeld, Germany) mounted on an upright Nikon FN-1 microscope (Düsseldorf, Germany). Wavelength was set to $740 \mathrm{~nm}$ and laser power to 200 to $300 \mathrm{~mW}$ at the objective output.

\section{Subcloning of H2B-PSmOrange and injection of mRNAs}

The pH2B-PSmOrange construct [29] (Addgene plasmid \#31920) was subcloned into the pCS2+ vector using the blunted XbaI restriction site. 260 pg H2B-PSmOrange mRNA were injected into one cell stage embryos. $80 \%$ ( $\mathrm{n}=150$ ) of injected zebrafish embryos showed strong nuclear H2B-PSmOrange protein expression for at least 4 days.

To label cell nuclei, $130 \mathrm{pg} H 2 A-C F P$ mRNA was injected.

\section{H2B-PSmOrange photoconversion and analysis}

For H2B-PSmOrange photoconversion, the photoconversion tool from the NIS-Elements AR software (Düsseldorf, Germany) was used: 17 to $20 \mathrm{~mW}$ of 488 
$\mathrm{nm}$ excitation laser power; $1 / 2$ scan speed frequency; 20 to 28 stimulation runs. $50 \mathrm{hpf}$ H2B-PSmOrange mRNA injected embryos were imaged before and after photoconversion using the sequential scanning mode for the 488, 561 and $637 \mathrm{~nm}$ channels. To determine the position of ThEPC cells, a z-stack was acquired at $4 \mathrm{dpf}$ with z-intervals of 1.0 to $2.0 \mu \mathrm{m}$.

The stacks were analyzed with our automatic ImageJ macro (Additional file 7). To highlight the regions of interest (ROIs), different automatic thresholds were applied in the green and far-red channel stacks after applying a 3D 'difference of Gaussians' filter to suppress noise and structures larger than the areas of interest. ROIs for each thresholded area were created with the Analyze Particles command, and overlapping ROIs identified and displayed in yellow.

\section{2-PM setup}

2-PM imaging and ablations were performed on a TriM Scope 2-photon microscope (LaVision BioTec GmbH, Bielefeld, Germany) mounted on a Nikon FN-1 upright stand (Düsseldorf, Germany) enclosed in a dark box, equipped with a femtosecond Ti:Sa laser (Chameleon Ultra II, Coherent, CA, USA), three PMTs (green channel: GaAsP, Hamamatsu; blue and red: standard, Hamamatsu, Herrsching am Ammersee, Germany) mounted on the ultrasensitive port next to the objective for increased sensitivity. Water-dipping objectives (16×, 0.8 numerical aperture (NA), long working distance (LWD), Nikon and 60×, 1.0 NA NIR Apo, Nikon, Düsseldorf, Germany) were used for image acquisition.

\section{Confocal laser-scan microscopy (CLSM) and image analysis}

For CLSM, embryos were embedded in 1.0\% low melting agarose in a glass bottom dish (MatTek, MA, USA or LabTek, CA, USA). Confocal images and stacks were acquired with a Nikon A1R (Düsseldorf, Germany) using a $20 \times$ air objective lens (NA 0.75); z-stack intervals were between $1.0 \mu \mathrm{m}$ and $2.0 \mu \mathrm{m}$.

$3 \mathrm{D}$ reconstructions, stack analysis and image adjustments were performed using the software Fiji, NIS-Element AR, Düsseldorf, Germany, Adobe Photoshop CS4, München, Germany and Image (NIH).

\section{Additional files}

Additional file 1: Movie S1. Long-term 2-PM time-lapse imaging reveals intermingling cells of different clusters, related to Figure 1. Dorsal view with anterior to the left, colour coded MIP obtained from a total zheight of $300 \mu \mathrm{m}$ focussed on the diencephalon in a Et(-1.0otpa:mmGFP) hd1 transgenic embryo. Time-lapse between $32 \mathrm{hpf}$ and $92 \mathrm{dpf}$. The LUT shows the $Z$ colour code table according to the depth of each stack. The stacks were acquired every 40 minutes with z-steps of $1.0 \mu \mathrm{m}$. Gamma was adjusted to a value of 0.70 for display purposes. White arrowheads mark the ThEPCs (arrows mark anterior ThEPC neurons), $\mathrm{IHb}$, rHb, and $\mathrm{dHb}$; red and green arrowheads highlight the second and the third cluster of projecting neurons, respectively. d, dorsal; Hb, habenula; IPN, interpeduncular nucleus; I, left; MIP, Maximum Intensity Projection; r, right; Tec, optic tectum; ThEPC, thalamic-epithalamic early projecting cluster.

Additional file 2: Movie S2. The ThEPCs are composed of mixed populations of dividing cells and postmitotic neurons, related to Figure 1. Dorsal view with anterior to the left, Colour Code MIP obtained from a total Z-height of $200 \mu \mathrm{m}$. High magnification time-lapse focussed on the ThEPC located in the left brain hemisphere between $32 \mathrm{hpf}$ and $52 \mathrm{hpf}$. The colour code spectrum table was used to highlight the depth of each stack. Stacks were acquired every 10 minutes with z-steps of $1.0 \mu \mathrm{m}$. Laser power correction was used to compensate for increasing depth (gamma $=0.60$ ). The manual tracking identifies dividing ThEPC cells, while other GFP positive neurons send out efferent projections. MIP, Maximum Intensity Projection; ThEPC, thalamic-epithalamic early projecting cluster.

Additional file 3: Figure S1. Ablation of $\mathrm{dHb}$ cells at $53 \mathrm{hpf}$, related to Figure 2.

Additional file 4: Figure S2. Ablation of ThEPC cells at $32 \mathrm{hpf}$, related to Figure 2.

Additional file 5: Figure S3. Photoconverted thalamic cells attach to the $\mathrm{dHb}$, related to Figure 3 .

Additional file 6: Movie S3. A population of ThEPC cells contribute to the $\mathrm{vHb}$ and is absent in embryos mutant for $t c f 7 \mid 2$, related to Figure 3. Dorsal view with anterior to the left, z-stacks of Et(-1.0otpa:mmGFP)hd1 and tcf712-/- x Et(-1.0otpa:mmGFP)hd1 transgenic embryos at $4 \mathrm{dpf}$ after photoconversion of the H2B-PSmOrange protein in the ThEPC region at 2 $\mathrm{dpf}$. Each stack was acquired using the sequential scanning mode with zsteps between $1.0 \mu \mathrm{m}$ and $2.0 \mu \mathrm{m}$. Gamma was adjusted for display purposes for each channel to values between 0.60 and 0.90. Colocalisation studies were performed using an automatic ImageJ macro for Fiji to identify the GFP positive, photoconverted ThEPC cells in the entire z-stack. The red channel is used to visualise the habenular morphology due to the nuclear expression of non-photoconverted H2B-PSmOrange protein at 4 dpf. Red ROls display the location of the photoswitched positive cells, while green ROls show the position of the GPF positive cells. Red and green ROls were combined to display the position of ThEPC cells expressing both GFP and the photoconverted protein in yellow. Minor corrections and compression were performed using the software iMovie and Wondershare Video Converter Ultimate. $\mathrm{Hb}$, habenula; $\mathrm{ROI}$, region of interest; ThEPC, thalamicepithalamic early projecting cluster; $v$, ventral.

Additional file 7: Automated macro for ImageJ (see Methods).

\section{Abbreviations}

2-PM: Two-photon microscopy; CLSM: Confocal laser-scan microscopy; dHb: Dorsal habenula; dpf: Days post fertilization; GFP: Green fluorescent protein; hpf: Hours post fertilization; IPN: Interpeduncular nucleus; LUT: Look up table; MIP: Maximum intensity projection; MR: Median raphe; PMT: Photomultiplier tube; ROI: Region of interest; Tcf: t-cell specific factor; ThEPC: Thalamic-epithalamic early projecting cluster; vHb: Ventral habenula.

\section{Competing interests}

The authors declare that they have no competing interests.

\section{Authors' contributions}

CB designed and performed all experiments. $C B$ and ND performed the time-lapse imaging and photoconversion experiments. PB developed the color code. MC designed and conceived the study and wrote the manuscript, which has been approved by all authors.

\section{Acknowledgements}

We thank many colleagues for discussion, plasmids and support, in particular J Wittbrodt, M Rembold, M Boutros, S Wilson, H Okamoto, O Subach, U Engel and members of our fish facility team for fish care. We are grateful to CellNetworks for funding of the 2-photon microscope and the Nikon Imaging Center at the University of Heidelberg for access to microscopy equipment and analysis software. Our work was supported by the Medical 
Faculty Mannheim to CB, the SFB488-Z3 to ND, and the DFG-SFB488-A18, DFG CA298/3-1 and the Wnt-Forschergruppe 1036/2 (AP-2) to MC.

\section{Author details}

'Department of Cell and Molecular Biology, Medical Faculty Mannheim, Heidelberg University, Ludolf-Krehl-Strasse 13-17, Mannheim 68167, Germany. ${ }^{2}$ Heidelberg University, COS and Nikon Imaging Center at the University of Heidelberg, Bioquant, Heidelberg 69120, Germany.

Received: 6 June 2013 Accepted: 23 July 2013

Published: 25 September 2013

\section{References}

1. Sutherland RJ: The dorsal diencephalic conduction system: a review of the anatomy and functions of the habenular complex. Neurosci Biobehav Rev 1982, 6:1-13.

2. Amo R, Aizawa H, Takahoko M, Kobayashi M, Takahashi R, Aoki T, Okamoto $\mathrm{H}$ : Identification of the zebrafish ventral habenula as a homolog of the mammalian lateral habenula. J Neurosci 2010, 30:1566-1574.

3. Gamse JT, Kuan YS, Macurak M, Brosamle C, Thisse B, Thisse C, Halpern ME: Directional asymmetry of the zebrafish epithalamus guides dorso-ventral innervation of the midbrain target. Development 2005, 132:4869-4881.

4. Aizawa H, Bianco IH, Hamaoka T, Miyashita T, Uemura O, Concha ML, Russell C, Wilson SW, Okamoto H: Laterotopic representation of left-right information onto the dorso-ventral axis of a zebrafish midbrain target nucleus. Curr Biol 2005, 15:238-243.

5. Bianco $\mathrm{H}$, Carl M, Russell C, Clark J, Wilson SW: Brain asymmetry is encoded at the level of axon terminal morphology. Neural Dev 2008, 3:9.

6. Yang L-M, Hu B, Xia Y-H, Zhang B-L, Zhao H: Lateral habenula lesions improve the behavioral response in depressed rats via increasing the serotonin level in dorsal raphe nucleus. Behav Brain Res 2008, 188:84-90.

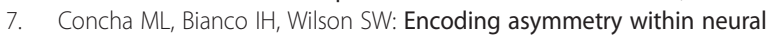
circuits. Nat Rev Neurosci 2012, 13:832-843.

8. Hikosaka O: The habenula: from stress evasion to value-based decisionmaking. Nat Neurosci 2010, 11:503-513.

9. Okamoto H, Agetsuma M, Aizawa H: Genetic dissection of the zebrafish habenula, a possible switching board for selection of behavioral strategy to cope with fear and anxiety. Dev Neurobiol 2012, 72:386-394.

10. Lecourtier L, Kelly PH: A conductor hidden in the orchestra? Role of the habenular complex in monoamine transmission and cognition. Neurosci Behav Rev 2007, 31:658-672.

11. Aizawa H, Goto M, Sato T, Okamoto H: Temporally regulated asymmetric neurogenesis causes left-right difference in the zebrafish habenular structures. Dev Cell 2007, 12:87-98.

12. Gamse JT, Thisse C, Thisse B, Halpern ME: The parapineal mediates leftright asymmetry in the zebrafish diencephalon. Development 2003, 130:1059-1068.

13. Concha ML, Wilson SW: Asymmetry in the epithalamus of vertebrates. J Anat 2001, 199:63-84.

14. Roussigne M, Blader P, Wilson SW: The zebrafish epithalamus clears a path through the complexity of brain lateralization. Dev Neurobiol 2011, 72:269-281.

15. Wree A, Zilles K, Schleicher A: Growth of fresh volumes and spontaneous cell death in the nuclei habenulae of albino rats during ontogenesis. Anat Embryol (Berl) 1981, 161:419-431.

16. Barth KA, Miklosi A, Watkins J, Bianco IH, Wilson SW, Andrew RJ: Fsi zebrafish show concordant reversal of laterality of viscera, neuroanatomy, and a subset of behavioral responses. Curr Biol 2005, 15:844-850.

17. Facchin L, Burgess HA, Siddiqi M, Granato M, Halpern ME: Determining the function of zebrafish epithalamic asymmetry. Philos Trans $R$ Soc Lond B Bio/ Sci 2009, 364:1021-1032.

18. Carl M, Bianco $\mid H$, Bajoghli B, Aghaallaei N, Czerny T, Wilson SW: Wnt/Axin1/betacatenin signaling regulates asymmetric nodal activation, elaboration, and concordance of CNS asymmetries. Neuron 2007, 55:393-405

19. Doll CA, Burkart JT, Hope KD, Halpern ME, Gamse JT: Subnuclear development of the zebrafish habenular nuclei requires ER translocon function. Dev Biol 2011, 360:44-57.

20. Roussigne $M$, Bianco $\Vdash H$, Wilson SW, Blader P: Nodal signaling imposes leftright asymmetry upon neurogenesis in the habenular nuclei. Development 2009, 136:1549-1557.
21. Bortolato M, Pivac N, Muck SD, Nikolac PM, Pessia M, Di Giovanni G: The role of the serotonergic system at the interface of aggression and suicide. Neuroscience 2013, 236:160-185.

22. Tsai SJ, Chiu HJ, Wang YC, Hong CJ: Association study of serotonin-6 receptor variant (C267T) with schizophrenia and aggressive behavior. Neurosci Lett 1999, 271:135-137.

23. Tsai SJ, Hong CJ, Yu YW, Lin CH, Song HL, Lai HC, Yang KH: Association study of a functional serotonin transporter gene polymorphism with schizophrenia, psychopathology and clozapine response. Schizophr Res 2000, 44:177-181.

24. Matsumoto M, Hikosaka O: Lateral habenula as a source of negative reward signals in dopamine neurons. Nat Geosci 2007, 447:1111-1115.

25. Matsumoto M, Hikosaka O: Representation of negative motivational value in the primate lateral habenula. Nat Neurosci 2009, 12:77-84.

26. Matsumoto M, Hikosaka O: Two types of dopamine neuron distinctly convey positive and negative motivational signals. Nat Geosci 2009, 459:837-841.

27. Bromberg-Martin ES, Hikosaka O: Lateral habenula neurons signal errors in the prediction of reward information. Nat Neurosci 2011, 14:1209-1216.

28. Zilles K, Schleicher A, Wingert F: Quantitative growth analysis of limbic nuclei areas fresh volume in diencephalon and mesencephalon of an albino mouse ontogenic series. J Hirnforsch 1976, 17:1-10.

29. Subach OM, Patterson GH, Ting L-M, Wang Y, Condeelis JS, Verkhusha W: A photoswitchable orange-to-far-red fluorescent protein, PSmOrange. Nat Methods 2011, 8:771-777.

30. Ekstrom P, Ebbesson SO: The left habenular nucleus contains a discrete serotonin-immunoreactive subnucleus in the coho salmon (Oncorhynchus kisutch). Neurosci Lett 1988, 91:121-125.

31. Bianco $\mathbb{H}$, Wilson SW: The habenular nuclei: a conserved asymmetric relay station in the vertebrate brain. Phil Trans R Soc B 2009, 364:1005-1020.

32. Kuan Y-S, Yu H-H, Moens CB, Halpern ME: Neuropilin asymmetry mediates a left-right difference in habenular connectivity. Development 2007, 134:857-865.

33. Snelson CD, Santhakumar K, Halpern ME, Gamse $\int \mathrm{T}$ : Tbx2b is required for the development of the parapineal organ. Development 2008, 135:1693-1702.

34. Concha ML, Russell C, Regan JC, Tawk M, Sidi S, Gilmour DT, Kapsimali M, Sumoy L, Goldstone K, Amaya E, et al: Local tissue interactions across the dorsal midline of the forebrain establish CNS laterality. Neuron 2003, 39:423-438

35. Heisenberg CP, Houart C, Take-uchi M, Rauch GJ, Young N, Coutinho P, Masai I, Caneparo L, Concha ML, Geisler R, et al: A mutation in the Gsk3binding domain of zebrafish Masterblind/Axin1 leads to a fate transformation of telencephalon and eyes to diencephalon. Genes Dev 2001, 15:1427-1434.

36. Hüsken U, Carl M: The Wnt/beta-catenin signaling pathway establishes neuroanatomical asymmetries and their laterality. Mech Dev 2013, 130:330-335.

37. Hagemann AlH, Scholpp S: The tale of the three brothers - Shh, Wnt, and Fgf during development of the thalamus. Front Neurosci 2012, 6:76.

38. Mattes B, Weber S, Peres J, Chen Q, Davidson G, Houart C, Scholpp S: Wnt3 and $\mathrm{Wnt} 3 \mathrm{a}$ are required for induction of the mid-diencephalic organizer in the caudal forebrain. Neural Dev 2012, 7:12.

39. Peukert D, Weber S, Lumsden A, Scholpp S: Lhx2 And Lhx9 determine neuronal differentiation and compartition in the caudal forebrain by regulating Wnt signaling. PLOS Biol 2011, 9(12):e1001218.

40. Ogawa S, Ramadasan PN, Goschorska M, Anantharajah A, Ng KW, Parhar IS: Cloning and expression of tachykinins and their association with kisspeptins in the brains of zebrafish. J Comp Neurol 2012, 520:2991-3012.

41. Muncan V, Faro A, Haramis A-PG, Hurlstone AFL, Wienholds E, van Es J, Korving J, Begthel H, Zivkovic D, Clevers H: T-cell factor 4 (Tcf7l2) maintains proliferative compartments in zebrafish intestine. EMBO Rep 2007, 8:966-973.

42. Young $R$, Reyes $A$, Allende M: Expression and splice variant analysis of the zebrafish tcf4 transcription factor. Mech Dev 2002, 117:269-273.

43. Clevers H, Nusse R: Wnt/b-catenin signaling and disease. Cell 2012, 149:1192-1205.

44. Beretta CA, Brinkmann I, Carl M: All four zebrafish Wnt7 genes are expressed during early brain development. Gene Expr Patterns 2011, 11:277-284.

45. Hansen T, Ingason A, Djurovic S, Melle I, Fenger M, Gustafsson O, Jakobsen KD, Rasmussen HB, Tosato S, Rietschel M, et al: At-risk variant in TCF7L2 for type II diabetes increases risk of schizophrenia. Biol Psychiatry 2011, 70:60-64. 
46. Alkelai A, Greenbaum L, Lupoli S, Kohn Y, Sarner-Kanyas K, Ben-Asher E, Lancet D, Macciardi F, Lerer B: Association of the type 2 diabetes mellitus susceptibility gene, TCF7L2, with schizophrenia in an Arab-Israeli family sample. PLoS One 2012, 7(1):e29228

47. Westerfield M: The zebrafish book. 3rd edition. Eugene, OR: The University of Oregon Press; 1995.

48. Macdonald R, Xu Q, Barth KA, Mikkola I, Holder N, Fjose A, Krauss S, Wilson SW: Regulatory gene expression boundaries demarcate sites of neuronal differentiation in the embryonic zebrafish forebrain. Neuron 1994, 13:1039-1053.

49. Shanmugalingam S, Houart C, Picker A, Reifers F, Macdonald R, Barth A, Griffin K, Brand M, Wilson SW: Ace/Fgf8 is required for forebrain commissure formation and patterning of the telencephalon. Development 2000, 127:2549-2561.

50. Kapsimali M, Kaushik AL, Gibon G, Dirian L, Ernest S, Rosa FM: Fgf signaling controls pharyngeal taste bud formation through miR-200 and DeltaNotch activity. Development 2011, 138:3473-3484.

doi:10.1186/1749-8104-8-19

Cite this article as: Beretta et al:: The ventral habenulae of zebrafish develop in prosomere 2 dependent on Tcf7l2 function. Neural

Development 2013 8:19.

\section{Submit your next manuscript to BioMed Central and take full advantage of:}

- Convenient online submission

- Thorough peer review

- No space constraints or color figure charges

- Immediate publication on acceptance

- Inclusion in PubMed, CAS, Scopus and Google Scholar

- Research which is freely available for redistribution 